\section{Acknowledgments}

The authors' work is supported by NCI grants U01 CA141541 and U01 CA141582 and by Athena Breast Health Network through the University of California Office of the President.

Address correspondence to: Robert D. Cardiff, Center for Comparative Medicine, UCD, Road 98 and Hutchison Drive, Davis, California 95616, USA. Phone: 530.752.2726; Fax: 530.752.7914; E-mail: rdcardiff@ucdavis.edu.
1. Santagata S, et al. Taxonomy of breast cancer based on normal cell phenotype predicts outcome. J Clin Invest. 2014;124(2):859-870

2. Rather LJ. The Genesis of Cancer: A Study in the History of Ideas. Baltimore, Maryland, USA: Johns Hopkins University Press; 1978.

3. Waldeyer HWG. Die Entwickelung der Carcinome. Virch Arch Pathol Anat. 1867;41:470-523.

4. Greenough RB, Taylor GW. Cancer of the breast, end results. N Engl J Med. 1934;210:831-835.

5. Macdonald I. Biological predeterminism in human cancer. Surg Gynecol Obstet. 1951;92(4):443-452.

6. Lerner BH. Great expectations: historical perspectives on genetic breast cancer testing. Am J Public Health. 1999;89(6):938-944.

7. Foote FW, Stewart FW. Lobular carcinoma in situ: a rare form of mammary carcinoma. Am J Surg Pathol. 1941;17(4):491-496.
8. Lerner BH. The Breast Cancer Wars: Hope, Fear, And The Pursuit Of A Cure In Twentieth-Century America. New York, New York, USA: Oxford University Press; 2001. 9. Tavassoli FA, Devilee P. International Agency for Research on Cancer., World Health Organization: Pathology and genetics of tumours of the breast and female genital organs. Lyon, France: IAPS Press; 2003.

10. Rakha EA, et al. Are triple-negative tumours and basal-like breast cancer synonymous? Breast Cancer Res. 2007;9(6):404.

11. Reis-Filho JS, Tutt AN. Triple negative tumours: a critical review. Histopathology. 2008;52(1):108-118.

12. Gusterson B. Do 'basal-like' breast cancers really exist? Nat Rev Cancer. 2009;9(2):128-134.

13. Moinfar F. Is 'basal-like' carcinoma of the breast a distinct clinicopathological entity? A critical review with cautionary notes. Pathobiology. 2008; 75(2):119-131.

\title{
An unexpected role for platelets in blocking Th17 differentiation
}

\author{
Ronjon Chakraverty
}

\begin{abstract}
Transplantation Immunology Group, Cancer Institute and Institute for Immunity and Transplantation, University College London, London, United Kingdom.
\end{abstract}
\begin{abstract}
It is well known that platelets interact with cells of the innate immune system to promote tissue repair. In contrast, it is less clear whether these links extend to cells of the adaptive immune system, such as $T$ cells. In this issue of the JCI, Morrell and colleagues provide compelling evidence that platelets are required to limit $\mathrm{CD} 4^{+} \mathrm{Th} 17$ differentiation through the actions of the chemokine platelet factor 4 (PF4). Absence of PF4 in the host leads to exaggerated Th17 differentiation after transplantation and rapid graft rejection. The authors' findings argue that platelets are not bit part players, but rather fully fledged, critical members of the adaptive immune system.
\end{abstract}

\section{Platelets as a bridge between coagulation and innate immunity}

In addition to their role in the hemostatic response to vascular injury, platelets provide an essential link to the innate immune system, enabling wound repair and reestablishing tissue homeostasis (1). Platelets are well positioned to act as a conduit between the coagulation and immune systems by providing a physical scaffold for cell interactions and delivering a large repertoire of immune mediators through exocytosis from storage granules or extrusion of membrane- and cytoplasm-containing microparticles (1). One of the most abundant platelet-derived immune molecules is the chemokine platelet factor 4 (PF4; also known as CXCL4). Upon platelet activation, $\mathrm{PF} 4$ is rapidly released from cytosolic $\alpha$ granules, leading to a huge increase in

Conflict of interest: The author has declared that no conflict of interest exists.

Citation for this article: J Clin Invest. 2014;

124(2):480-482. doi:10.1172/JCI74231. local concentration (2). The functions of PF4 are complex, generating both pro- and anticoagulant actions (3) and differentially affecting multiple cell types, including megakaryocytes, endothelial cells, and innate immune cells (2-4). For example, in response to endothelial injury, plateletderived PF4 is crucial for monocyte recruitment, survival promotion, and proinflammatory macrophage differentiation (5). PF4 also binds other chemokines, such as CXCL8 (6) and CCL5 (5), to generate heteromers with distinct activities. Together, the diverse actions of PF4 pose an enormous challenge to investigators attempting to understand its physiological functions.

The interaction between platelets and a damaged endothelium is a common feature of many disorders in humans. In the chronic inflammatory lesions associated with atherosclerosis, PF4 is critical for macrophage infiltration and disease progression (5). Endothelial injury may also occur in transplanted organs, either as a result of the effects of ischemia and reperfusion or as the result of antibody and cellular responses within the host (7). Furthermore, platelets have been proposed to be important mediators of the initial host response to grafted tissue, but it is not clear how platelets contribute to the ensuing adaptive immune response.

\section{Loss of PF4 accelerates graft rejection by enhancing Th17}

In this issue of the JCI, Shi, Morrell, and colleagues examined how platelets and platelet-derived PF4 affect anti-donor T cell responses after transplantation (8). Based on the broad premise that platelets and PF4 induce a proinflammatory response, the authors hypothesized that deletion of Pf4 in host mice would protect MHC class II-mismatched cardiac allografts from rejection. In this murine model of cardiac transplant, graft rejection is usually mediated by classical Th1-type responses, where $\mathrm{CD}^{+} \mathrm{T}$ cell effectors produce cytokines such as IFN- $\gamma$, IL-2, and GM-CSF (9). Although Th1 differentiation was disabled in $\mathrm{Pf}^{-/-}$hosts, Morrell and colleagues surprisingly found that grafts in these mice were rejected at an earlier time point compared with control animals (8). Histological examination of rejected grafts from $\mathrm{Pf}^{-/-}$mice revealed an atypical vasculopathy, with perivascular aggregates composed of $\mathrm{T}$ cells and dense infiltrates of neutrophils. Similar neutrophil recruitment after transplantation has been previously observed in Tbx $21^{-/-}$mice, 
which lack TBET, a key regulator of Th1 differentiation. Tbx $21^{-/-}$mice also exhibit accelerated graft rejection as the result of a shift from a Th1-type response toward a Th17-type response (9). Th17 effectors are usually required to clear extracellular bacteria and fungi by indirectly activating neutrophils through production of the cytokines IL-17A and IL-17F; however, Th17 effectors have also been implicated in the pathogenesis of several autoimmune disorders (10). Early Th17 developmental programs require the integrated actions of STAT3, which promotes expression of Rorc (encoding retinoic acid-related orphan receptor $\gamma t$ [ROR $\gamma t])$, the "master regulator" of Th17 effectors (10). In the present study, graft-associated cellular infiltrates in $\mathrm{Pf}^{-/-}$hosts were heavily skewed toward a Th17 gene signature, as opposed to the Th1-associated signature observed in control mice (8). Treatment of $\mathrm{Pf}^{-/-}$mice with anti-IL-17 antibodies prevented accelerated graft rejection, which indicates that PF4 negatively regulates a functionally relevant Th17 response.

Although publicly available databases suggest that steady-state PF4 expression is confined primarily to megakaryocytelineage cells and monocytes, Morrell and colleagues demonstrated that $\mathrm{CD}^{+} \mathrm{T}$ cells also expressed PF4 after transplantation (8), raising the possibility that $\mathrm{PF} 4$ regulation of $\mathrm{CD}^{+}$Th17 differentiation is cell autonomous. However, in a series of elegant chimera experiments, in which PF4 expression was confined to individual cellular compartments, the authors demonstrated that while T cell-derived PF4 had a minor role in repressing Th17 programming, it was more likely that platelet-derived PF4 was required to prevent an inappropriate Th17 response after transplant. Additionally, Th17 skewing was observed in thrombopoietin receptor-deficient mice, which have low numbers of platelets and low levels of plasma $\mathrm{PF} 4$, but produce $\mathrm{T}$ cell-derived PF4.

\section{PF4 influences $\mathrm{T}$ cell programming}

The findings of Morrell and colleagues provide impetus for further examination of $\mathrm{PF} 4$ and $\mathrm{CD}^{+} \mathrm{T}$ cell programming, particularly in diseases in which endothelial injury accompanies adaptive immunity. Several pieces of the puzzle still remain, including where and how PF4 signaling modulates $\mathrm{T}$ cells. Because platelets are largely absent from lymph nodes, it seems probable that the interaction between platelet-derived PF4 and T cells occurs during the effector phase, when $\mathrm{CD}^{+} \mathrm{T}$ cells would have access to adherent platelets on damaged endothelia or interact directly with PF4 deposited in the form of microvesicles within tissue (11). Although in vitro studies suggested that soluble or platelet-derived PF4 could act upon $\mathrm{CD}^{+}$ $\mathrm{T}$ cells in cis (8), it is unclear whether this interaction requires a specific $T$ cell-associated receptor or is mediated indirectly by the capacity of PF4 to bind multiple cell surface glycosaminoglycans. A splice variant of human CXCR3 binds PF4 with high affinity (12), but it is not known whether this isoform is present in mice. However, additional experiments by Morrell and colleagues suggested that administration of antibodies targeting CXCR3 after transplantation did not recapitulate the accelerated rejection seen in $\mathrm{Pf}^{-/-}$mice (8).

This study provides some clues as to how PF4 influences Th17 lineage specification. While Th1 and Th2 have distinct differentiation programs, Th17 developmental programs overlap with those of other $\mathrm{CD}^{+}$ $\mathrm{T}$ cell subsets, including induced Tregs (iTregs) (10). Both Th17 cells and iTregs require TFG- $\beta$ signaling during the early phase of lineage commitment, and here the authors found that PF4 directly blocked TFG- $\beta$ signaling in primary $\mathrm{T}$ cells (8). Consistent with PF4-dependent inhibition of TGF- $\beta$ signaling, both Th17- and iTreg-associated transcripts were increased in grafts of $\mathrm{Pf}^{-{ }^{--}}$recipients. It is unlikely that $\mathrm{PF} 4$-associated TFG- $\beta$ interference alone can explain repression of Th17 responses. Early iTreg developmental programs induced by TGF- $\beta$ are relatively plastic and prone to Th17 deviation. For example, iTregs exposed to proinflammatory cytokines that activate STAT3 during early development can skew toward Th17 (10); therefore, it also will be important to consider how these factors influence PF4 actions. Further analysis will require systematic dissection of the effects of PF4 on T cells, at the level of chromatin structure as well as DNA binding of lineagespecifying transcription factors. The mechanisms for PF4 suppression of Th17 cells are likely both complex and context dependent, as suggested by the observation that PF4 deficiency enhanced Th17 differentiation after transplantation, but not after malarial parasite infection (8).

\section{Disease implications and future directions}

Notably, Morrell and colleagues found that thrombopoietin receptor-deficient mice also had an increased propensity for Th17 responses after transplantation (8). Recent studies in patients with autoimmune conditions associated with reduced numbers of platelets (and, presumably, PF4) also exhibit increased Th17 cell frequencies $(13,14)$. In patients with immune thrombocytopenia, an aberrant host immune response targets platelets for peripheral destruction, and this loss of platelets is associated with increases in both Th1 and Th17 cells (14). Similarly, Th17 responses have been linked to aplastic anemia development in animal models and in humans (13). One possibility for both immune thrombocytopenia and aplastic anemia is that reductions in platelet mass and $\mathrm{PF} 4$ promote an increase in Th17 cells, which occurs as a secondary event that is not reflective of the primary immune pathophysiology. Alternatively, PF4 deficiency may reinforce immunopathology by driving Th17 differentiation and contributing to disease progression. It is also possible that Th17 development in response to reductions in PF4 contribute to development of graft-versus-host disease (GVHD) after allogeneic hematopoietic stem cell transplantation. In GVHD, donor $\mathrm{T}$ cells are activated and undergo effector differentiation after transplant, when platelet numbers are low. Although involvement of Th17 cells in acute GVHD is controversial, Th17 cells appear to contribute to skin- and lung-associated manifestations of chronic GVHD (15), where disease severity inversely correlates with platelet numbers (16).

Perhaps the most exciting implication of the study of Morrell and colleagues is the prospect that PF4 or its derivatives could be used therapeutically to ameliorate Th17driven autoimmune disorders or to prevent transplant rejection (8). Because PF4 can facilitate both pro- and antiinflammatory immune responses, it is unlikely that use of unmodified PF4 will translate to the clinic. Furthermore, in order for a PF4-dependant therapeutic approach to be effective, combinatorial strategies that block the entire Th1-Th17 inflammatory axis, rather than Th17 differentiation alone, would need to be developed. This study raises the prospect that improved understanding of PF4 actions may help to identify small-molecule inhibitors of Th17 developmental programs.

\section{Acknowledgments}

R. Chakraverty receives funding from Leukaemia and Lymphoma Research, United Kingdom. 
Address correspondence to: Ronjon Chakraverty, Transplantation Immunology Group, Cancer Institute and Institute for Immunity and Transplantation, University College London, London, United Kingdom. Phone: 44.207.472.6100; Fax: 44.207.830.2092; E-mail: r.chakraverty@ucl.ac.uk.

1. Rondina MT, Weyrich AS, Zimmerman GA. Platelets as cellular effectors of inflammation in vascular diseases. Circ Res. 2013;112(11):1506-1519.

2. Sachais BS, Higazi AA, Cines DB, Poncz M, Kowalska MA. Interactions of platelet factor 4 with the vessel wall. Semin Thromb Hemost. 2004;30(3):351-358.

3. Kowalska MA, Rauova L, Poncz M. Role of the platelet chemokine platelet factor 4 (PF4) in hemostasis and thrombosis. Thromb Res. 2010;125(4):292-296.

4. Lambert MP, Rauova L, Bailey M, Sola-Visner MC, Kowalska MA, Poncz M. Platelet factor 4 is a negative autocrine in vivo regulator of megakaryopoi- esis: clinical and therapeutic implications. Blood. 2007;110(4):1153-1160.

5 . Koenen RR, et al. Disrupting functional interactions between platelet chemokines inhibits atherosclerosis in hyperlipidemic mice. Nat Med. 2009; 15(1):97-103.

6. Dudek AZ, Nesmelova I, Mayo K, Verfaillie CM, Pitchford S, Slungaard A. Platelet factor 4 promotes adhesion of hematopoietic progenitor cells and binds IL-8: novel mechanisms for modulation of hematopoiesis. Blood. 2003;101(12):4687-4694.

7. Baldwin WM 3rd, Kuo HH, Morrell CN. Platelets: versatile modifiers of innate and adaptive immune responses to transplants. Curr Opin Organ Transplant. 2011;16(1):41-46.

8. Shi G, et al. Platelet factor 4 limits Th17 differentiation and cardiac allograft rejection. J Clin Invest. 2014;124(2):543-552.

9. Yuan X, et al. A novel role of CD4 Th17 cells in mediating cardiac allograft rejection and vasculopathy. J Exp Med. 2008;205(13):3133-3144.

10. Basu R, Hatton RD, Weaver CT. The Th17 family: flexibility follows function. Immunol Rev. 2013; 252(1):89-103.

11. Boilard E, et al. Platelets amplify inflammation in arthritis via collagen-dependent microparticle production. Science. 2010;327(5965):580-583.

12. Lasagni $L$, et al. An alternatively spliced variant of CXCR3 mediates the inhibition of endothelial cell growth induced by IP-10, Mig, and I-TAC, and acts as functional receptor for platelet factor 4. J Exp Med. 2003;197(11):1537-1549.

13. de Latour RP, et al. Th17 immune responses contribute to the pathophysiology of aplastic anemia. Blood. 2010;116(20):4175-4184.

14. Johnsen J. Pathogenesis in immune thrombocytopenia: new insights. Hematology Am Soc Hematol Educ Program. 2012;2012:306-312.

15. Serody JS, Hill GR. The IL-17 differentiation pathway and its role in transplant outcome. Biol Blood Marrow Transplant. 2012;18(1 suppl):S56-S61.

16. Akpek G, et al. Development of a prognostic model for grading chronic graft-versus-host disease. Blood. 2001;97(5):1219-1226.

\title{
Lipids rule: resetting lipid metabolism restores T cell function in systemic lupus erythematosus
}

\author{
Yoko Kidani ${ }^{1}$ and Steven J. Bensinger ${ }^{1,2,3,4}$
}

\begin{abstract}
1Department of Pathology and Laboratory Medicine, ${ }^{2}$ Department of Molecular and Medical Pharmacology, and ${ }^{3} U C L A$ Metabolomics Center, David Geffen School of Medicine, UCLA, Los Angeles California, USA. ${ }^{4}$ Jonsson Comprehensive Cancer Center, UCLA, Los Angeles California, USA.
\end{abstract}

\begin{abstract}
Systemic lupus erythematosus (SLE) is a devastating autoimmune disease characterized by chronic inflammation and systemic destruction of host organs or tissue. A key feature of SLE is T cell dysfunction characterized by hyperresponsive antigen receptor signaling. In this issue of the JCI, McDonald and colleagues provide evidence that homeostasis of a subset of lipids, the glycosphingolipids (GSLs), is severely perturbed in the membranes of $T$ cells from SLE patients. Furthermore, normalization of GSLs restored TCR signaling and ameliorated $T$ cell dysfunction. These data suggest that targeting host metabolism may be an effective means of reinforcing selftolerance and attenuating autoimmunity.
\end{abstract}

Systemic lupus erythematosus (SLE) is a systemic autoimmune disease characterized by immune hyperactivity and loss of immunologic tolerance to self-antigens. Clinical manifestations often include symptoms of chronic inflammation, such as fatigue and fevers, as well as more specific features, including skin rashes, renal disease, arthritis, cardiovascular disease, vasculopathies, coagulopathies, and CNS involvement. The etiology of SLE is not well understood and likely includes both environmental and genetic factors. However, it is clear that dysfunction of multiple facets of host immunity underlies SLE pathogen-

Conflict of interest: The authors have declared that no conflict of interest exists.

Citation for this article: $J$ Clin Invest. 2014;

124(2):482-485. doi:10.1172/JCI74141 esis, resulting in inflammatory immune cell infiltrates, autoantibody production, and deposition of pathogenic antibodies in target organs (reviewed in ref. 1).

\section{Lipid dysfunction \\ in autoimmune disease}

Lipid dysfunction is a common clinical observation in many patients with rheumatic diseases and results in a heightened risk of cardiovascular disease independent of therapy (2-4). Genetic deletion of key proteins, such as apolipoprotein E, involved in lipid homeostasis has also been shown to exacerbate SLE pathogenesis in a broad array of model systems (5-7). Furthermore, T cells isolated from SLE patients have intrinsic alterations in lipid composition, especially in specialized microdomains of the plasma membrane (also known as lipid rafts) that contain the $\mathrm{T}$ cell antigen receptor (TCR), and alterations in associated signaling molecules $(8,9)$. The combined weight of these observations argues that lipid metabolism influences self-tolerance and autoimmune pathogenesis; however, it is not known how defects in lipid metabolic programs direct the fate and function of immune cells in autoimmunity. In this issue, McDonald and colleagues shed new light on the complex crosstalk between lipid metabolism and $\mathrm{T}$ cell dysfunction by demonstrating that resetting glycosphingolipid (GSL) homeostasis partially restores TCR signaling and normalizes function in $\mathrm{T}$ cells isolated from patients with lupus (10).

\section{Alterations in $\mathrm{T}$ cell responsiveness associated with SLE}

It has long been appreciated that $\mathrm{T}$ cells purified from individuals with SLE are dysfunctional, particularly in patients with active disease (reviewed in ref. 1). Perhaps the best-characterized changes in $\mathrm{T}$ cells from SLE patients are profound alterations in TCR signaling. Detailed studies indicate that $\mathrm{T}$ cells from SLE patients display heightened calcium flux in response to antigen receptor stimulation. The molecular mechanisms underlying the acquisition of this "exaggerated" signaling phenotype 